\title{
Factors Affecting the Length of Hospitalization after Laparoscopic Resection of Gastric Subepithelial Tumor
}

\author{
Yeon Soo Chang, M.D., Ph.D. \\ Department of Surgery, Eulji Medical Center, Eulji University College of Medicine, Seoul, Korea
}

\begin{abstract}
A subepithelial tumor (SET) is a common disease of the stomach. A gastrointestinal stromal tumor (GIST), which has malignant potential, is the most common tumor among SETs. The definite treatment of SETs of the stomach should be a complete resection; thus, a laparoscopic wedge resection has been the standard treatment for SET of the stomach. The surgical and oncologic outcomes of laparoscopic wedge resections for gastric GIST have shown it to be safe and feasible compared to open surgery. The authors reported that small sized SET and tumor located in areas other than the posterior wall can be a candidate for shorter hospitalization. The critical pathway (CP) and new therapeutic options can be helpful for reducing the hospital stay. On the other hand, it is important not to overlook the fatal complications after discharge.
\end{abstract}

Keywords: Laparoscopy, Stomach, Gastrointestinal stromal tumor, Hospitalization

\author{
Received August 31, 2019 \\ Revised September 6, 2019 \\ Accepted September 6, 2019 \\ Corresponding author \\ Yeon Soo Chang \\ Department of Surgery, Eulji Medical \\ Center, Eulji University College of \\ Medicine, 68 Hangeulbiseok-ro, \\ Nowon-gu, Seoul 01830, Korea \\ Tel: +82-2-970-8688 \\ Fax: +82-2-970-8227 \\ E-mail: cutdowngs@naver.com \\ ORCID: \\ https://orcid.org/0000-0002-6570-5920
}

Copyright ( 2019 The Journal of Minimally Invasive Surgery. All rights reserved.

This is an Open Access article distributed under the terms of the Creative Commons Attribution Non-Commercial License (http:/ creativecommons.org/licenses/by-nc/4.0/) which permits unrestricted non-commercial use, distribution, and reproduction in any medium, provided the original work is properly cited.

Subepithelial tumors (SETs) are a common disease of the stomach. An endoscopic biopsy for a pathological diagnosis is difficult because SETs are usually covered with normal mucosa. Gastrointestinal stromal tumors (GISTs), which have malignant potential regardless of their size, are the most common tumors among SETs of the stomach. Therefore, definite treatment of a SET of the stomach should be a complete resection. A local resection is regarded as the primary treatment because lymph node metastasis is quite rare in GIST.

With the development of laparoscopic instruments and surgical techniques, a laparoscopic wedge resection has been the standard treatment for SET of the stomach. The surgical and oncologic outcomes of a laparoscopic wedge resection for gastric GIST have shown it to be safe and feasible compared to open surgery, even in large GIST over $5 \mathrm{~cm}$ in size., ${ }^{2,3}$

The advantages of laparoscopic surgery over open surgery are well known, including shorter hospitalization and rapid recovery to usual activity. The authors analyzed the factors influencing the morbidity and hospital stay after a laparoscopic resection of gastric SET. The results showed that a larger size $(>5 \mathrm{~cm})$ and location on the posterior wall of the stomach can prolong the hospital stay than expected. The authors report that a small sized SET and tumor located other than the posterior wall can be a candidate for shorter hospitalization. Stanek et al. ${ }^{3}$ reported similar results in that the hospital stay was longer in the larger tumor group $(>5 \mathrm{~cm})$. A laparoscopic resection of SET in a difficult location of the stomach is quite a challenge. An inadequate angle for the laparoscopic approach and insufficient exposure of the tumor can increase the postoperative morbidity, including leakage, bleeding, and stenosis. On the other hand, another report showed no difference in the hospital stay in different anatomical locations of gastric 
GIST. ${ }^{4}$

The hospital stay can be affected by not only the size and location of the tumor, but also by many other factors, including fever, pain, wound infection, oliguria, hemorrhage, and general condition of the patient. Like the results of the authors of this article, a well-configured critical pathway (CP) standardizes the various treatment protocols to reduce the variables during hospitalization and medical costs and increase patient satisfaction.

Recently, an emerging therapeutic option for gastric SET has been introduced. Laparoscopic and endoscopic cooperative surgery (LECS) as well as non-exposed endoscopic wallinversion surgery (NEWS) have advantages for the precise localization and resection of small-sized gastric SETs, particularly in endophytic tumors. ${ }^{5,6}$ A robotic-assisted resection can be quite helpful in gastric SET in unfavorable locations in a manner that provides adequate exposure, resection, and repair with articulating instruments. These new options can be expected to help reduce the hospital stay.

Short-term hospitalization has many advantages, but it is important to prepare safe treatment protocols and to check all conditions of the patients before discharge so that fatal complications are not overlooked after discharge.

\section{ORCID}

Yeon Soo Chang, https://orcid.org/0000-0002-6570-5920

\section{CONFLICT OF INTEREST}

None.

\section{FUNDING}

None.

\section{ACKNOWLEDGMENTS}

None.

\section{REFERENCES}

1) Akahoshi K, Oya M, Koga T, Shiratsuchi Y. Current clinical management of gastrointestinal stromal tumor. World J Gastroenterol 2018;24:2806-2817.

2) Yang Z, Li P, Hu Y. Laparoscopic versus open wedge resection for gastrointestinal stromal tumors of the stomach: a meta-analysis. Wideochir Inne Tech Maloinwazyjne 2019;14:149-159.

3) Stanek M, Pisarska M, Rzepa A, Radkowiak D, Major P, Budzynski A. Laparoscopic treatment of large gastrointestinal stromal tumors (> 5 cm). Wideochir Inne Tech Maloinwazyjne 2019;14:170175.

4) Chen Q, Lin J, Zheng C, et al. [Impact of laparoscopic surgery on efficacy in the treatment of gastrointestinal stromal tumors in different anatomical locations]. Zhonghua Wei Chang Wai Ke Za Zhi 2016;19:1277-1281.

5) Wang H, Cao L, Zheng K, Zhao Y. Laparoscopic Endoscopic Cooperative Surgery for Gastrointestinal Stromal Tumors. Surg Laparosc Endosc Percutan Tech 2018;28:354-358.

6) Aoyama J, Goto O, Kawakubo H, et al. Clinical outcomes of nonexposed endoscopic wall-inversion surgery for gastric submucosal tumors: long-term follow-up and functional results. Gastric Cancer 2019 Jul 5 [Epub]. DOI:10.1007/s10120-019-00985-1.

7) Arseneaux M, Yarbrough D, Nagamoto T. Robotic-assisted freehanded, full-thickness gastric GIST resection with primary repair in unfavorable locations. J Robot Surg 2019;13:491-494. 